\title{
Influência do tratamento específico na prevalência de enteroparasitoses e esquistossomose mansônica em escolares do município de Barra de Santo Antônio, AL
}

\author{
Influence of specific treatment of intestinal parasites and schistosomiasis \\ on prevalence in students in Barra de Santo Antônio, AL \\ Gilberto Fontes ${ }^{1}$, Karyna Karla Lessa Oliveira', Alessandra Karine Lessa Oliveira ${ }^{1}$ \\ e Eliana Maria Mauricio da Rocha ${ }^{1}$
}

\begin{abstract}
Resumo De 1.020 estudantes submetidos a exame coproparasitológico (método de Lutz), 938 (92\%) apresentaram positividade para pelo menos uma espécie de enteroparasito. Seis meses pós-tratamento, $383(37,5 \%)$ estudantes foram reavaliados e $347(90,6 \%)$ apresentaram exames positivos. Não houve diferença significativa nas prevalências antes e após tratamento específico, entretanto poliparasitismo foi significativamente menor na segunda análise.
\end{abstract}

Palavras-chaves: Parasitas intestinais. Exame coproscópico. Helmintos. Epidemiologia.

\begin{abstract}
From a total of 1,020 students examined, 983 (92\%) showed positive results for at least one species of parasite. Six months after treatment, a sample of 383 students (37.5\%) was reevaluated and $347(90.6 \%)$ presented positive results. There was no significant change in prevalence before and after treatment, although the number of individuals that had multiple parasitism was significantly lower in the second sample.
\end{abstract}

Key-words: Intestinal parasites. Surveys. Helminths. Epidemiology.

No Brasil, principalmente na região Nordeste, as enteroparasitoses ainda são muito comuns devido, sobretudo, ao saneamento básico deficiente e à precária educação sanitária da população ${ }^{1}$. Dentre as parasitoses, a esquistossomose mansônica tem grande importância devido à alta morbi-mortalidade $e$ sua prevalência se mantém elevada em algumas regiões, inclusive em áreas urbanas do Nordeste do Brasil ${ }^{7}$. Segundo a Organização Mundial de Saúde, os programas de controle de parasitoses devem dar prioridade às crianças em idade escolar, que representam o grupo vulnerável de crescimento mais rápido nos países endêmicos ${ }^{7}$.

O objetivo desta investigação foi determinar a prevalência das enteroparasitoses e esquistossomose mansônica em uma amostra aleatória de escolares e também analisar o efeito do tratamento específico na prevalência das infecções helmínticas.

O estudo foi realizado entre agosto de 1998 e dezembro de 1999 no município de Barra de Santo Antônio, litoral norte de Alagoas, situado a $45 \mathrm{~km}$ de Maceió, com população de 11.351 habitantes (9.574 na área urbana), em área de $138 \mathrm{~km}^{2}$ e 2.326 domicilios $^{3}$. Foram examinados escolares da $1^{\text {a }}$ a $4^{\text {a }}$ séries do 1으 grau, das oito escolas da rede pública local. As amostras fecais, conservadas em solução de formol a $10 \%$, foram examinadas pelo método de Lutz (sedimentação espontânea) ${ }^{4}$. Os examinados não haviam usado previamente anti-hemínticos e os parasitados detectados recebiam tratamento

\footnotetext{
1. Departamento de Patologia do Centro de Ciências Biológicas da Universidade Federal de Alagoas, Maceió, AL.

Financiamento: Fundação de Amparo a Pesquisa do Estado de Alagoas (FAPEAL)

Este estudo foi aprovado pelo Comitê de Bioética da UFAL/CEP (processo $n^{\circ} 23065.001375$ ).

Endereço para correspondência: Prof. Gilberto Fontes. Dept ${ }^{\circ}$ Patologia/Centro de Ciências Biológicas/UFLA. Praça Afrânio Jorge s/n, 57010-020 Maceió, $\mathrm{AL}$, Brasil.

Tel: 5582 327-3336; Fax: 5582 221-2501

e-mail: gf@fapeal.br

Recebido para publicação em 15/7/2003

Aceito em 22/7/2003
} 
específico, com orientação e acompanhamento médico (mebendazol, metronidazol, praziquantel). Seis meses após o tratamento, uma amostra aleatória dos escolares previamente examinados foi reavaliada e os que apresentaram parasitoses foram novamente tratados. $O$ tamanho das amostras foi calculado usando o pacote estatístico EPI INFO, baseado em estudo piloto, aceitando $\alpha=0,05$ e considerando $15 \%$ o erro máximo tolerável.

De 1.356 escolares matriculados, 1.222 (90,1\%) assistiram palestras educativas e 1.020 (83,5\%) submeteram-se ao exame coproparasitológico. Desses, $938(92 \%)$ tiveram exame positivo para pelo menos uma espécie de parasita, sendo que 171 (18,2\%) apresentaram monoparasitismo e 767 (81,8\%) poliparasitismo com até 8 diferentes espécies. Os exames coproparasitológicos revelaram 11 diferentes espécies de parasitas na população em estudo (Tabela 1); freqüência antes do tratamento. Os casos de esquistossomose mansônica eram todos autóctones. Os parasitas mais freqüentes foram os geohelmintos

\begin{tabular}{|c|c|c|c|c|c|}
\hline \multirow[b]{2}{*}{ Parasitas } & \multicolumn{2}{|c|}{ Freqüência $A T^{\mathrm{b}}(\mathrm{n}=1.020)$} & \multicolumn{2}{|c|}{ Freqüência $\mathrm{PT}^{\mathrm{c}}(\mathrm{n}=383)$} & \multirow[b]{2}{*}{$\mathrm{p}^{\mathrm{d}}$} \\
\hline & $\mathrm{n}^{\circ}$ & $\%$ & $\mathrm{n}^{\circ}$ & $\%$ & \\
\hline Trichuris trichiura & 603 & 59,1 & 153 & 39,9 & $0,0001^{e}$ \\
\hline Ascaris lumbricoides & 581 & 57,0 & 146 & 38,1 & $0,0001^{\mathrm{e}}$ \\
\hline Ancilostomatidae & 523 & 51,3 & 118 & 30,8 & $0,0001^{e}$ \\
\hline Giardia lamblia & 275 & 27,0 & 207 & 54,0 & $0,0001^{e}$ \\
\hline Entamoeba coli & 272 & 26,7 & 106 & 27,7 & 0,70 \\
\hline Entamoeba histolytica & 202 & 19,8 & 89 & 23,2 & 0,16 \\
\hline Strongyloides stercoralis & 143 & 14,0 & 37 & 9,7 & 0,03 \\
\hline Enterobius vermicularis & 25 & 2,5 & 6 & 1,6 & 0,31 \\
\hline Hymenolepis nana & 25 & 2,5 & 4 & 1,0 & 0,10 \\
\hline Schistosoma mansoni & 24 & 2,4 & 4 & 1,0 & 0,12 \\
\hline Taenia sp & 14 & 1,4 & 4 & 1,0 & 0,63 \\
\hline Enteroparasitoses geral $^{a}$ & 938 & 92,0 & 347 & 90,6 & 0,41 \\
\hline \multicolumn{6}{|c|}{ a Inclui os casos de poliparasitismo. } \\
\hline \multicolumn{6}{|c|}{ b AT = antes do tratamento específico. } \\
\hline \multicolumn{6}{|c|}{${ }^{\mathrm{c}} \mathrm{PT}=$ pós-tratamento específico } \\
\hline \multicolumn{6}{|c|}{${ }^{d} p$ - comparação prevalência AT e PT } \\
\hline${ }^{\mathrm{e}} \mathrm{p}<0,05$ (diferença estatis & te signif & & & & \\
\hline
\end{tabular}

Trichuris trichiura, Ascaris lumbricoides e Ancilostomatidae, mas a prevalência de parasitas transmitidos pelo contato com água, como protozoários, também foi elevada. Dos parasitados, 44,7\% eram masculinos e $55,3 \%$ femininos ( $p>0,05)$. A idade dos examinados variou de 5 a 18 anos, tendo os parasitados $10,7 \pm 2,3$ anos e os negativos $9,8 \pm 2,5$ anos $(p<0,05)$. Dos 1.020 examinados inicialmente, uma amostra aleatória de 383 (37,5\%), entre parasitados ou não previamente, foi reavaliada seis meses após tratamento específico. Destes, 347 (90,6\%) apresentaram exames positivos para algum tipo de parasita (Figura 1), sendo identificadas 11 espécies de enteroparasitas (Tabela 1): freqüência póstratamento. Parasitas que apresentaram índices superiores a $50 \%$ de prevalência antes do tratamento, reduziram significativamente a freqüência após tratamento. Os demais permaneceram com taxas semelhantes antes e após tratamento, com exceção da Giardia, que teve a prevalência aumentada (Tabela 1). Dentre os parasitados após tratamento, $92(26,5 \%)$ apresentaram monoparasitismo e 255 (73,5\%) poliparasitismo com até seis espécies diferentes. Apesar de não haver diferença significativa nas prevalências antes e após o tratamento $(p>0,05)$, poliparasitismo foi significativamente maior na primeira amostra (Odds relativa $=1,62$; I.C. 95\%: $1,20-2,19$ ). Os resultados sugerem que o tratamento foi eficaz para reduzir o grau de parasitismo no período, mas não contribuiu para a eliminação das enteroparasitoses, visto que o risco de infecção permaneceu o mesmo. Análise de trabalhos recentes no Brasil, mesmo entre populações indígenas, onde prevalências de enteroparasitoses são reconhecidamente elevadas ${ }^{5}$, não se detecta taxas tão altas como as encontradas em Barra de S. Antônio. A infestação por enteroparasitas está claramente relacionada com a pobreza e precariedade das condições de higiene e saneamento ${ }^{6}$, características da área urbana estudada, onde apenas 1,2\% dos domicílios possuem rede de esgoto, $76,7 \%$ são servidos por abastecimento de água de rede geral e $61,6 \%$ dos domicílios apresentam destino de lixo coletado ${ }^{3}$. Além disso, o município apresenta baixo nível educacional e sócio-econômico, sendo a taxa de alfabetização $59,1 \%{ }^{3}$. Isso, aliado às precárias condições de saneamento contribuem provavelmente para a alta prevalência de enteroparasitas observada, como também para a freqüente ocorrência 


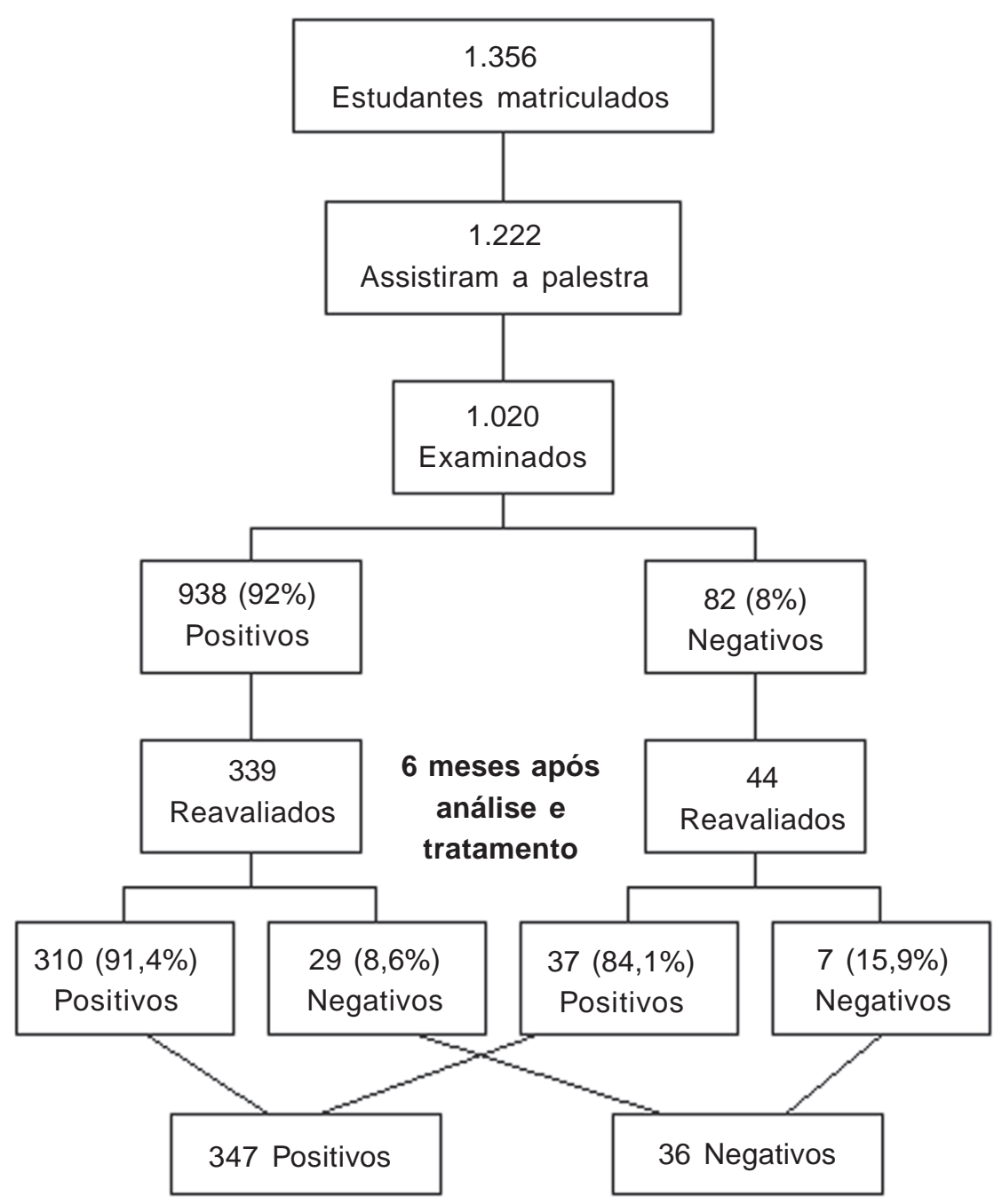

Figura 1- Esquema do estudo com as amostras dos escolares examinados nas fases pré e pós-tratamento específico para helmintíases intestinais e esquitossomose mansônica.

de poliparasitismo. A baixa positividade de Schistosoma mansoni, Strongyloides stercoralis, Enterobius vermicularis e Taenia $s p$ deve ser vista com cautela, uma vez que o método de diagnóstico empregado não é o mais adequado para detectar esses parasitas, sugerindo que esses helmintos, possivelmente têm prevalências maiores que as aqui relatadas.

Há muito se salienta que as parasitoses intestinais representam um grave problema de ordem sanitária e social e que são necessárias medidas combinadas de terapêutica, saneamento básico e conscientização para que se obtenha efetivo controle dessas enfermidades. De fato, Ferreira et $\mathrm{al}^{2}$ atribuem a redução na prevalência das parasitoses em geral, observada em São Paulo, de 30,9\% em 1984 para $10,7 \%$ em 1996, a melhorias no abastecimento de água e no esgotamento sanitário. Apenas o tratamento dos parasitados não resulta na diminuição da prevalência, como observado no presente estudo. É fundamental o estabelecimento de uma política de saúde que não seja baseada só no diagnóstico e tratamento individual, mas que vise a eliminação das fontes de infecção. Portanto, o controle dessas parasitoses passa por melhorias de condições sócio-econômicas, saneamento básico e na educação sanitária da população, fatores quase que inexistentes na comunidade em estudo. 


\section{REFERÊNCIAS BIBLIOGRÁFICAS}

1. Amaral RS, Camillo-Coura L, Pimenta Jr FG, Coelho GE, Loiola, CC. Epidemiología $Y$ control de las geohelmintiasis en Brasil. Reunión sobre el Control de las Helmintiasis Intestinales en el Contexto de AIEPI: Informe, Rio de Janeiro, Brasil. Organización Panamericana de la Salud, Organización Mondiale del Salud, p. 39-43, 1998.

2. Ferreira MU, Ferreira CS, Monteiro CA. Tendência secular das parasitoses intestinais na infância na cidade de São Paulo (1984-1996). Revista de Saúde Pública 34:73-82, 2000.

3. Fundação Instituto Brasileiro de Geografia e Estatística. Instituto Brasileiro de Geografia e Estatística. In: XII Recenseamento Geral Brasileiro, (Alagoas, Censo Demográfico, Dados Distritais), 2000.
4. Lutz AV. Schistosoma mansoni e Schistosomose, segundo observações feitas no Brasil. Memórias do Instituto Oswaldo Cruz 11:121-125, 1919.

5. Miranda RA, Xavier FB, Menezes RC. Parasitismo intestinal em uma aldeia indígena Parakanã, sudeste do estado do Pará, Brasil. Cadernos de Saúde Pública 14:507-511, 1998.

6. Tavares-Dias M, Grandini AA. Prevalência e aspectos epidemiológicos de enteroparasitoses na população de São José da Bela Vista, São Paulo. Revista da Sociedade Brasileira de Medicina Tropical 32:63-65, 1999.

7. World Health Organization. The Control of Schistosomiasis. $2^{\text {nd }}$ report of the WHO Expert Commitee on Schistosomiasis. Tecnical Report Series, nº. 830, 110p, 1993. 